\title{
Storming Majorana's Tower with OAM states of light in a plasma
}

\author{
F. TAMBURINI ${ }^{1,2}$ and B. ThidÉ ${ }^{3,4}$ \\ 1 Department of Astronomy, University of Padua, vicolo dell' Osservatorio 3, IT-331 22 Padua PD, Italy, EU \\ 2 Associazione CIVEN, via delle Industrie 5, TORRE HAMMON, IT-30175 Venezia-Marghera, Italy, EU \\ ' 3 Swedish Institute of Space Physics, Ångström Laboratory, P. O. Box 537, SE-75121 Uppsala, Sweden, EU \\ 4 Galilean School of Higher Education, University of Padua, Via Giovanni Prati 19, IT-351 22 Padua PD, Italy, EU
}

PACS 42.50.Tx - Optical angular momentum and its quantum aspects

PACS 03.65.-w - Quantum mechanics

PACS 14.70.Bh - Photons

\begin{abstract}
We extend the relationship between mass and spin angular momentum, described by the bosonic spectrum of positive definite mass particles of the Majorana solution to the Dirac equation, to photons that acquire an effective Proca mass through the Anderson-Higgs mechanism 1] when they propagate in a plasma. In an earlier paper 2] we showed that if the plasma is structured, it can impart orbital angular momentum (OAM) to the photons that reduces the total Proca photon mass. Here we show, through a generalisation of Majorana's solution, that photons with OAM in a plasma cannot assume negative squared mass states. This means that there exist interesting analogies with Quantum Gravity or General Relativity models involving a modified action of the Lorentz group.
\end{abstract}

Introduction. - In 1932, Majorana [3] formulated an alternative solution to the Dirac equation for bosonic and fermionic relativistic particles with null or positive definite rest mass whose particle spectrum is also known 'as the "Majorana Tower" 4. This particular solution was formulated in an attempt to avoid the problem of the negative squared mass solution emerging from the relativistic electron wavefunction, which led to the concepts of anti-electron and anti-matter, identified experimentally by C. D. Anderson [5. The particle spectrum described by the Majorana solution is characterized by a specific mass/spin relationship that does not possess any correspondence with the spectrum of known elementary particles in the Standard Model [6]. Instead, a subset of the Majorana particle spectrum describes a particular class of fermions, the so-called Majorana neutrinos. These fermions are supposed to be found in neutrino-less double beta decays through a lepton-number violating nuclear decay process [7] $]$. In the spectrum of Majorana's solution, a particle and its antiparticle are not mutually distinguishable [10.

Photons can in all aspects be considered to be Majorana particles. They carry energy, linear momentum and angular momentum and have, in free space, zero rest mass. Their total angular momentum $\mathbf{J}$ is the sum of the spin angular momentum (SAM) $\mathbf{S}$ and the orbital angular mo- mentum (OAM) L. From a classical point of view, the electromagnetic field can carry linear momentum as well as angular momentum all the way to infinity [11,12] and therefore these physical observables can be utilised for wireless information transfer over long distances [13, 14].

A photon propagating in a plasma acquires an effective mass $m^{*}$ due to its interaction with plasmons; this effect is described by the Anderson-Higgs mechanism [1,15] or as a hidden gauge invariance of the Proca-Maxwell equations 16. If the plasma is homogeneous and characterized by its unperturbed plasma frequency $\omega_{p 0}$, and if the perturbation induced by the photons in the plasma is neglected, the mass acquired by photons will be $m^{*}=\hbar \omega_{p 0} / c^{2}$, where $\hbar$ and $c$ are the reduced Planck constant and the speed of light, respectively. In order to simplify the notation, we will henceforth in this Letter use natural units, $e=c=\hbar=G=1$, so that the virtual photon mass in an unperturbed, homogeneous plasma coincides with the unperturbed plasma frequency.

Recently it was shown theoretically that a photon propagating in a structured plasma will acquire OAM and an associated additional effective mass [2. This is because the OAM acts as an effective mass-reducing term. An electromagnetic wave traversing a plasma introduces a perturbation in the plasma particle distribution, assumed to comprise electrons and heavy, immobile positive 
ions providing a neutralising background. The square of the plasma frequency becomes $\omega_{p}^{2}(x)=\omega_{p 0}^{2}(1+\eta(r, \varphi, z))$, where $\eta(r, \varphi, z)$ is the electron distribution perturbation. Then the following relationship between the acquired effective Proca mass $\mu_{\gamma}$ and the perturbation in the motion of the electrons caused by the electromagnetic (EM) wave associated with the photon holds:

$$
\mu_{\gamma}=\frac{1}{1+\frac{\hat{\mathbf{v}} \cdot \nabla \Phi}{E}}\left(\omega_{p}^{2}-4 \pi \frac{n(r) \delta \dot{v}-\hat{\mathbf{v}} \cdot \square \nabla \Phi}{E}\right)^{1 / 2}
$$

Here, $\hat{\mathbf{v}}=\mathbf{v} /|v|$ denotes the unit direction vector of the electron velocity in the plasma, $\mathbf{v}$, assumed, in accordance with Ref. 2, to be parallel to the electric field $\mathbf{E}$, and $\delta \dot{v}=\hat{\mathbf{v}} \cdot \partial_{t} \mathbf{v}=\left|\partial_{t} v\right|$.

The EM wave propagating in the plasma is assumed to generate a weak perturbation $\delta \mathbf{v}$ of the unperturbed electron velocity vector $\mathbf{v}_{0}$ such that the perturbed velocity is $\mathbf{v}=\mathbf{v}_{0}+\delta \mathbf{v}$, and higher order corrections can be neglected. Let us consider the simple case of a helicoidal plasma density perturbation $\tilde{n} \cos \left(\ell \varphi+q_{0} z\right)$ where $q_{0}$ is the helix step. For the OAM value $\ell$ in the Proca squared mass term, we then find that

$$
\begin{aligned}
& \mu_{\gamma}^{2}= \frac{E}{E+\hat{\mathbf{v}} \cdot \nabla \Phi} \omega_{p 0}^{2}(1+\eta(r, \varphi, z)) \\
&-\frac{1}{E+\hat{\mathbf{v}} \cdot \boldsymbol{\nabla} \Phi}\left(4 \pi \delta \dot{v}\left(n_{0}+\tilde{n} \cos \left(\ell \varphi+q_{0} z\right)\right)\right. \\
&\quad-4 \pi \mathbf{v} \cdot \square \nabla \Phi)
\end{aligned}
$$

where $n_{0}$ is the unperturbed electron density and $m_{e}$ the electron mass. The geometry of the perturbation is described in cylindrical coordinates, $\mathbf{r} \equiv(r, \varphi, z)$ and the spiral formed plasma perturbation winds along the $z$ axis, along which the EM wave is propagating. Of course, one can describe the case of a more general perturbation $f(\ell, q)$ as a superposition of helicoidal perturbations. It is interesting to note that a similar behaviour is found in the London equations that describe fields and currents in superconductors. Also there, a characteristic length that defines the behaviour of EM field is present 17. The London penetration depth $\lambda_{L}$ is the characteristic length of penetration of a magnetic field in a superconductor that depends on mass, density and charge of the electrons in the super-conducting medium similarly to what is seen in equation (2).

The term in equation (2) containing the photon OAM $\ell_{0}$ acts as a negative squared mass term for the virtual (Proca) photon mass, exhibiting the possibility of generating a photon with negative squared mass, as expected in certain Lorentz and gauge symmetry violations [18 21.

By extending the Majorana solution to the Dirac equation in free space to photons in a plasma carrying OAM, we show in this Letter that the Proca mass of a photon in a plasma is always positive definite when Lorentz invariance is preserved.
Space-time symmetries. - In the Proca-Maxwell equations, Lorentz invariance is preserved but the gauge invariance is lost because both the vector and scalar potentials of the EM field, A and $\Phi$, respectively, become at all effects observable quantities. The EM energy density, $u$, depends explicitly on the potentials and, consequently, on the energy densities $\mu_{\gamma}^{2} \Phi^{2} / 8 \pi$ and $\mu_{\gamma}^{2} \mathbf{A}^{2} / 8 \pi$ in the following way:

$$
u=\frac{1}{8 \pi}\left(\mathbf{E}^{2}+\mathbf{B}^{2}+\mu_{\gamma}^{2} \Phi^{2}+\mu_{\gamma}^{2} \mathbf{A}^{2}\right) .
$$

Following the Majorana-Oppenheimer formulation of QED 22, 23, we represent the photon wavefunction in Riemann-Silberstein (RS) formalism, in which the wavefunction is described by the complex RS vector $\mathbf{G}=\mathbf{E} \pm \mathrm{iB}[12,24,26$. The photon obeys a Dirac-like equation with the well-known problems of photon localisability 12, 27,29. Without loss of generality for any particular choice of helicity state or multiplicative constants, the Maxwell equations in free space become

$$
\begin{gathered}
\mathrm{i} \boldsymbol{\nabla} \times \mathbf{G}= \pm \frac{\partial \mathbf{G}}{\partial t} \\
\nabla \cdot \mathbf{G}=0
\end{gathered}
$$

By using the correspondence principle $\mathbf{p} \leftrightarrow \hat{\mathbf{p}} \equiv-\mathrm{i} \boldsymbol{\nabla}$, equation (4a) leads to a Dirac-like wave equation

$$
\mp \mathrm{i} \frac{\partial}{\partial t} \mathbf{G}+\mathrm{i} \hat{\mathbf{p}} \times \mathbf{G}=\mathbf{0}
$$

whereas equation (4b) describes the transversality of the EM fields with respect to the propagation direction, namely, $\hat{\mathbf{p}} \cdot \mathbf{G}=0$.

Let us consider the infinitesimal Lorentz transformations described by the $4 \times 4$ matrix sets $\mathbf{S}=\left\{\mathrm{S}_{x}, \mathrm{~S}_{y}, \mathrm{~S}_{z}\right\}$ and $\mathbf{T}=\left\{\mathbf{T}_{x}, \mathbf{T}_{y}, \mathbf{T}_{z}\right\}$, where the set elements are

$$
\begin{aligned}
\mathrm{S}_{x} & =\left(\begin{array}{cccc}
0 & 0 & 0 & 0 \\
0 & 0 & 0 & 0 \\
0 & 0 & 0 & -1 \\
0 & 0 & 1 & 0
\end{array}\right) \\
\mathrm{S}_{y} & =\left(\begin{array}{cccc}
0 & 0 & 0 & 0 \\
0 & 0 & 0 & 1 \\
0 & 0 & 0 & 0 \\
0 & -1 & 0 & 0
\end{array}\right) \\
\mathrm{S}_{z} & =\left(\begin{array}{cccc}
0 & 0 & 0 & 0 \\
0 & 0 & -1 & 0 \\
0 & 1 & 0 & 0 \\
0 & 0 & 0 & 0
\end{array}\right)
\end{aligned}
$$


and

$$
\begin{aligned}
& \mathrm{T}_{x}=\left(\begin{array}{llll}
0 & 1 & 0 & 0 \\
1 & 0 & 0 & 0 \\
0 & 0 & 0 & 0 \\
0 & 0 & 0 & 0
\end{array}\right) \\
& \mathrm{T}_{y}=\left(\begin{array}{llll}
0 & 0 & 1 & 0 \\
0 & 0 & 0 & 0 \\
1 & 0 & 0 & 0 \\
0 & 0 & 0 & 0
\end{array}\right) \\
& \mathrm{T}_{z}=\left(\begin{array}{llll}
0 & 0 & 0 & 1 \\
0 & 0 & 0 & 0 \\
0 & 0 & 0 & 0 \\
1 & 0 & 0 & 0
\end{array}\right)
\end{aligned}
$$

respectively. By taking the $3 \times 3$ spatial parts, one defines the sub-matrices $\mathbf{s}=\left(\mathrm{s}_{x}, \mathrm{~s}_{y}, \mathrm{~s}_{z}\right)$ of the $\mathbf{S}$ matrices, and by writing the Hamiltonian of the Dirac-like equation for the photon as (cf. Refs. 12, 27 and 30 32)

$$
\hat{\mathrm{H}}= \pm \mathbf{s} \cdot \hat{\mathbf{p}}
$$

one obtains

$$
\mathrm{i} \frac{\partial}{\partial t} \mathbf{G}=\hat{\mathrm{H}} \mathbf{G} .
$$

The Hamiltonian $\hat{\mathbf{H}}$ has eigenvalues $\pm p, 0$; the eigenvalue 0 is forbidden by the transversality condition [33].

The Majorana Tower. - Let us now in more detail analyse the Majorana formulation of the infinite-spin component Dirac equation with positive definite real mass eigenvalues and in the absence of an external field [3, also known as the "Majorana Tower" of particles [4. Here, because of the chosen space-time symmetries of the Lorentz group applied to the Dirac equation in free space, the mass spectra of bosons and fermions always assume positive definite values and follow a precise mass/spin angular momentum relationship.

A particular case is the class of zero rest mass particles such as photons, neutrinos and gravitons that differ from their antiparticles only because of their helicity state 222; they belong to a wider class of inequivalent relativistic representations of massless vector fields with arbitrary helicity states 34 .

Let us consider the particular case of the EM field. Conformal invariance and the postulate of the vector character of the EM field imply that part of these general fields do admit Maxwell's equations as a special case. In these terms, each of these more general fields can be described by a set of matrices $S_{\mu \nu}$ in a vectorial representation of the generators $D(0,1)$ and $D(1,0)$, or by the equivalent spinorial representations of the generators $D(1 / 2,0)$ and $D(0,1 / 2)[22$.

We write the general Dirac equation in the form

$$
\left[\gamma_{0}+W+\alpha \cdot \hat{\mathbf{p}}-\beta \mu_{\gamma}\right] \mathbf{G}=0
$$

where $\gamma_{0}, \alpha$ and $\beta$ are the usual Dirac matrices, $\mu_{\gamma}$ is the mass of the particle and $W$ the energy $[3,35,36$. As is well known, the Dirac equation for the relativistic electron admits solutions with both positive and negative mass eigenvalues.

Let us apply the Majorana formalism to the photon in a perturbed plasma. When a quantum of light is propagating in a region filled with plasma, it acquires a positive virtual mass for both helicity states of the wave equation. For this reason, the eigenvalues of the matrix $\beta$ of equation (10) and the quantity $\tilde{\mathbf{G}} \beta \mathbf{G}$ must be positive definite. Through non-unitary transformations one can, according to Ref. 3, write the quantity $\tilde{\mathbf{G}} \beta \mathbf{G}=\tilde{\mathbf{g}} \mathbf{g}$ of the RS vector and obtain an equivalent formulation

$$
\left(\gamma_{0}+W+\gamma \cdot \hat{\mathbf{p}}-\mu_{\gamma}\right) \mathbf{g}=0
$$

where the invariance of the function $\tilde{\mathbf{g} g}$ is provided by unitary transformations 3 .

From the infinitesimal Lorentz transformations, equations (6) and (7), one writes a set of Hermitean operators, $a_{j}=\mathrm{iS}_{j}$ and $b_{j}=-\mathrm{i} \top_{j}$, for the $j$ th space-time coordinate. The commutation rules then become

$$
\begin{array}{ll}
{\left[a_{x}, a_{y}\right]=\mathrm{i} a_{z},} & {\left[b_{x}, b_{y}\right]=-\mathrm{i} a_{z}} \\
{\left[a_{x}, b_{x}\right]=0,} & {\left[a_{x}, b_{y}\right]=\mathrm{i} b_{z}, \quad\left[a_{x}, b_{z}\right]=\mathrm{i} b_{y}}
\end{array}
$$

The spectrum of these particles exhibits a relationship between the intrinsic spin angular momentum, $s$, with another parameter, $m^{*}$, the "Majorana-mass" term, which is related to the particle's rest mass or to the virtual mass. This property is valid for both bosonic $(j=1,2,3, \ldots)$ and fermionic $(j=1 / 2,3 / 2,5 / 2, \ldots)$ solutions,

$$
\mu_{\gamma}=\frac{m^{*}}{s+1 / 2}
$$

and describes a spectrum cast in an infinite tower of particles with positive mass values that decrease when the intrinsic angular momentum of the particle increases.

Space-time symmetries and plasma. - We now show that photons in a structured plasma follow a similar mass/angular momentum relationship, with the difference that the mass term is given by the Proca mass and the spin angular momentum $\mathbf{S}$ is replaced by the total angular momentum, $\mathbf{S} \rightarrow \mathbf{J}=\mathbf{L}+\mathbf{S}$.

During the propagation of a light beam, the total angular momentum $\mathbf{J}$ is conserved 12 . When photons traverse a structured inhomogeneous medium, angular momentum conversions occur with the result of generating beams of light carrying OAM. According to Ref. 2, one finds that the higher the value of the OAM acquired by the photon, the lower the total effective Proca mass of the particle, analogously to what is described with spin angular momentum and mass for a Majorana particle. More precisely, in the Proca mass formulation, the unperturbed squared mass quantity, $m^{* 2}$, becomes that of the perturbed plasma, $\mu_{\gamma}^{2}$, given by equation (2). 
In the Proca mass term the OAM term $\ell$ induced by the plasma structure becomes evident with the characteristic scale $q_{0}$,

$$
\mu_{\gamma}=\omega_{p 0} A \sqrt{1-\frac{4 \pi \delta \dot{v}}{E+\hat{\mathbf{v}} \cdot \nabla \Phi} \frac{n_{0}+\tilde{n} f(\ell, q)}{\omega_{p 0}^{2} A^{2}}}
$$

After some algebra, one finds that the Proca mass equations leads to an approximate mass/angular momentum relationship that extends the vacuum (free space) solution proposed by Majorana:

$$
\mu_{\gamma}=\frac{E+\hat{\mathbf{v}} \cdot \nabla \Phi}{\omega_{p 0} A(E+\hat{\mathbf{v}} \cdot \nabla \Phi)+4 \pi \delta \dot{v}\left(n_{0}+\tilde{n} f(\ell, q)\right)}
$$

This expression can be written in a form that easily recalls the original Majorana formula of equation (13),

$$
\mu_{\gamma}=\frac{m^{*}}{\Sigma(\ell, q)+1 / 2}
$$

where $\Sigma(\ell, q)$ is a general function of the OAM of the photon and of the characteristic spatial scale of the perturbation that summarises all the terms present in equation (15), and the Majorana mass term coincides with that acquired by the photon in the unperturbed plasma.

Unlike the mathematical structure of the space-time manifold described by the Lorentz group, in which space is homogeneous and isotropic and time homogeneous, a plasma may exhibit spatial/temporal structures that break the space-time symmetry. The inhomogeneity of plasma structures and the interaction between photons and charges in the plasma actually change the Proca mass turning time invariance into a more complicated space/time invariance, with the result of converting part of the Proca mass into orbital angular momentum. Moreover, the mathematical correspondence between the Majorana solution and the behaviour of a photon in a plasma confirms the Ansatz that OAM states cannot induce a negative squared mass to the photon, if Lorentz invariance is to be preserved. Alternatively, one can prove this conjecture by following the relationship between Proca mass and OAM in Ref. 2 in the simplest case of an helicoidal perturbation: the mass squared term of the photon is positive if the Proca mass is larger than the absolute value of the term containing OAM,

$$
\begin{aligned}
E \omega_{p 0}^{2}(1+\eta(r, \varphi, z)) & +4 \pi \hat{\mathbf{v}} \cdot \square \nabla \Phi \\
& >4 \pi \delta \dot{v}\left(n_{0}+\tilde{n} \cos \left(\ell \varphi+q_{0} z\right)\right)
\end{aligned}
$$

which is found to be always valid, in agreement with the hypothesis of small perturbation assumed in our calculations.

The Majorana Tower, derived from infinitesimal Lorentz transformations, allows also solutions for which the energy $W$ and momentum p obey a tachyonic relationship, for which $W= \pm \sqrt{c^{2} p^{2}-k^{2} c^{4}}$. This solution, which can be interpreted in terms of Lorentz invariance violation [37, is beyond the scope of the present Letter.
Conclusions. - Photons that propagate in a structured plasma acquire mass and orbital angular momentum because of the hidden gauge invariance due to the Anderson-Higgs mechanism in Proca-Maxwell equations. The squared-mass term that describes photon OAM acts as a negative squared mass term that could apparently cause photons to acquire a negative squared mass. We find, however, that the mass/total angular momentum relationship of Proca-Maxwell equations forbids the negative squared mass condition.

The presence of the plasma, and the spatial structuring with characteristic scale length $q$ induce a mass/total angular momentum relationship with the properties of the spin/mass relationship expected for massive Majorana particles. The infinite spin Majorana's solution to the Dirac equation gives, by definition, a spectrum of particles with a positive definite or null finite mass. The difference between the original solution proposed by Majorana, based on space-time symmetries of the Lorentz group applied to the Dirac equation in free space, and that of photons in a structured plasma lies in the contribution of the OAM to the mass/angular momentum relationship in this latter case.

The presence of a characteristic scale length and structures in the plasma, as expected in nature, shows clear analogies with models of space-time involving modified action of the Lorentz group, such as the Magueijo-Smolin model, in which there exists an energy scale that acts as invariant [38]. Moreover, the mathematical structure found in our work shows deep analogies with the dynamics described by the Dirac equation in the presence of a characteristic scale that unavoidably introduces Berry phase effects 39].

This type of solution could find interesting applications in the propagation of light and radio waves in plasma, in plasma physics and more generally in studies of the propagation of photons in nonlinear inhomogeneous media. Our result can be applied to other particles such as neutrinos, involving spinor fields, and gravitons giving a new perspective to the meaning of the Majorana Tower.

The extension of the Majorana Tower to gravitons is not so immediate. In fact, until now, no theory of quantum gravity exists, and no gravitational waves have been directly observed. The coupling of gravitons and the plasma frequency is not known and could have a different strength and even lead to imaginary effective masses, which would correspond to the impossibility of screening gravitational waves with plasma.

\section{REFERENCES}

[1] Anderson P. W., Phys. Rev., 130 (1963) 439.

[2] Tamburini F., Sponselli A., Thidé B. and Mendonca J. T., Europhys. Lett., 90 (2010) 45001.

[3] Majorana E., Nuov. Cim., 9 (1932) 335.

[4] Magueijo J., A Brilliant Darkness (Basic) 2010.

[5] Anderson C. D., Phys. Rev., 43 (1933) 491. 
[6] Hagiwara K. et al., Phys. Rev. D, 66 (2002) 010001 Review of Particle Properties.

[7] Doi M., Kotani T. and Takasugi E., Prog. Theor. Phys. Suppl., 83 (1985) 1.

[8] Mohapatra R. N. et al., Rep. Prog. Phys., 70 (2007) 1757.

[9] Avignone, ili F. T., Elliott S. R. and Engel J., Rev. Mod. Phys., 80 (2008) 481.

[10] Majorana E., Nuov. Cim., 14 (1937) 171.

[11] Jackson J. D., Classical Electrodynamics 3rd Edition (Wiley \& Sons, New York, NY, USA) 1999.

[12] ThidÉ B., Electromagnetic Field Theory 2nd Edition (Dover Publications, Inc., Mineola, NY, USA) 2011. http://www.plasma.uu.se/CED/Book

[13] Gibson G., Courtial J., Padgett M. J., Vasnetsov M., Pas'ko V., Barnett S. M. and Franke-Arnold S., Opt. Express, 12 (2004) 5448.

[14] Tamburini F., Mari E., Thidé B., Barbieri C. and Romanato F., Appl. Phys. Lett., (99) (2011) 204102.

[15] Schwinger J., Phys. Rev., 125 (1962) 397.

[16] Mendonça J. T., Theory of Photon Acceleration (IOP Publishing, Bristol, UK) 2001.

[17] Tinkham M., Introduction to Superconductivity (Dover Publications) 2004.

[18] Rühl W., Nuov. Cim., 68 (1970) 235.

[19] Goldhaber A. S. and Nieto M. M., Rev. Mod. Phys., 43 (1971) 277.

[20] Goldhaber A. S. and Nieto M. M., Rev. Mod. Phys., 82 (2010) 939.

[21] Ferrero A. and Altschul B., Phys. Rev. D, 80 (2009) 125010 .

[22] Tamburini F. and Vicino D., Phys. Rev. A, 78 (2008) 052116(5).

[23] Giannetto E., Lett. Nuov. Cim., 44 (1985) 140.

[24] Silberstein L., Ann. Phys. (Leipzig), 327 (1907) 579.

[25] Silberstein L., Ann. Phys. (Leipzig), 329 (1907) 783.

[26] Berry M. V., J. Opt. A: Pure Appl. Opt., 6 (2004) S175.

[27] Mignani R., Recami E. and Baldo M., Lett. Nuov. Cim., 11 (1974) 568.

[28] Bialynicki-Birula I., Acta Phys. Polon. A, 86 (1994) 97.

[29] Bialynicki-Birula I., Phys. Rev. Lett., 80 (1998) 5247.

[30] Bialynicki-Birula I., Photon wave function in Prog. Opt., edited by Wolf E., Vol. XXXVI (Elsevier, Amsterdam, Holland) 1996 pp. 245-294.

[31] Bialynicki-Birula I., The photon wave function in proc. of Coherence and Quantum Optics VII, edited by EBERLY J. H., Mandel L. and Wolf E., (Plenum, New York) 1996 pp. 313-322.

[32] Kobe D. H., Found. Phys., 29 (1999) 1203.

[33] Berestetski V. B., Lifshitz E. M. and Pitaevski L. P., Quantum Electrodynamics second, reprinted Edition (Pergamon Press, Oxford, UK) 1989.

[34] Fushchich V. I. and Nikitin A. G., Symmetries of Maxwell's Equations (D. Reidel, Dordrecht, Holland) 1987.

[35] Dirac P. A. M., Proc. Roy. Soc. A, 117 (1928) 610.

[36] Thaller B., The Dirac Equation 1st Edition (Springer) 2010.

[37] TAmburini F. and LAVEDER M., Arxiv preprint arXiv:1109.5445, (2011).

[38] Magueijo J. and Smolin L., Phys. Rev. Lett., 88 (2002)
190403.

[39] Gosselin P., Bérard A., Mohrbach H. and Ghosh S., Phys. Lett. B, 660 (2008) 267. 